\title{
Two-dimensional imaging of a helicon discharge
}

\section{David D Blackwell and Francis F Chen}

\author{
Electrical Engineering Department, University of California, Los Angeles, \\ CA 90095-1594, USA
}

Received 21 March 1997, in final form 10 September 1997

\begin{abstract}
Two-dimensional images of the ionized argon light of a helicon discharge are made both along and across the magnetic field with various antenna configurations. Two antennas, a Nagoya type III and a helical antenna, are used to create a magnetized RF plasma with density in the range $n \leq 5 \times 10^{13} \mathrm{~cm}^{-3}$. A CCD camera with a $488 \mathrm{~nm}$ bandpass filter is used to image the plasma as magnetic field and power are changed. By inserting a Faraday shield, it is demonstrated that the inductive component of the antenna coupling is responsible for producing high-density plasmas. Asymmetries in the plasma profile are shown to be caused primarily by capacitive coupling, with the purely inductively coupled plasmas being symmetric and centrally peaked. Numerical calculations of antenna coupling show that the configurations having the largest antenna loading correspond to the brightest plasmas observed in the experiment, with the $m=+1$ mode being the most strongly coupled.
\end{abstract}

\section{Introduction}

Over the past decade there has been increasing interest in helicon wave discharges because they have been shown [1-7] to produce high-density plasmas $\left(n>10^{12} \mathrm{~cm}^{-3}\right)$ with relatively small amounts of RF power $\left(P_{r f} \leq 3 \mathrm{~kW}\right)$. Theoretical treatments $[8,9]$ of this discharge have been done using the well developed theory of waves in a bounded plasma, giving several scaling laws to which experimental data are fitted. Unfortunately, many observations have been made $[10,11]$ which are not easily explained, and clearly depend at least partially on antenna coupling. Only recently [12-15] have complete numerical methods been developed to properly solve the problem of antenna coupling, allowing a more realistic comparison of theory and experiment to be made.

With a view toward such experiments, we examine in this paper the experimental conditions required to match the assumptions in the theories. In particular, we can observe the effect of capacitive coupling, which is usually neglected in theory, by inserting a Faraday shield between the antenna and the glass chamber. It is shown that even in high-density regimes where the plasma has been referred to as 'wave coupled' [10], capacitive coupling can have a significant effect on the plasma properties. Most apparent was the effect of this coupling on the plasma profile, which shows that using simple helicon wave theory to explain the observed profiles [16] is in most cases unsatisfactory. We show that previously observed jumps in density [5] as the magnetic field or RF power is raised cannot be described simply by the density on axis. The entire density profile changes at the jumps. A second important parameter is antenna geometry. In previous experiments, more than one antenna configuration is used $[3,6,11]$, but until now the antenna coupling calculations were not easily available, so that interpretation of results could only be qualitative. Here, we compare our observations with the results from a numerical code which solves the problem of antenna coupling to a nonuniform plasma [15]. Specifically, we show that an $m=+1$ helical antenna yields a higher-density plasma than a straight Nagoya type III antenna, in agreement with the predictions of the code. This agreement, along with the effects of eliminating the capacitive coupling, demonstrates that the inductive coupling of the antenna to helicon waves is responsible for producing high plasma densities.

\section{Apparatus}

The plasma was created in a $108 \mathrm{~cm}$ long, $10 \mathrm{~cm}$ diameter Pyrex chamber (figure 1), which is evacuated to a base pressure $p \leq 3 \times 10^{-6}$ Torr and surrounded by eight $25 \mathrm{~cm}$ diameter coils which produce a magnetic field in the range $350-1000 \mathrm{G}$. The centre of the antenna is located approximately $25 \mathrm{~cm}$ from the axial midplane of the chamber, at which point there are two ports located $90^{\circ}$ azimuthally from each other. One port is for a Langmuir probe, and the other is used for the gas feed and pressure monitors. The antenna is driven with $P_{r f} \leq 2 \mathrm{~kW}$ of RF power at $13.56 \mathrm{MHz}$ in $50 \mathrm{~ms}$ pulses about $4 \mathrm{~s}$ apart. A standard $L$-type capacitive tuning circuit is used to match the power supply to the antenna, and the incident and reflected powers are monitored with in-line power meters 


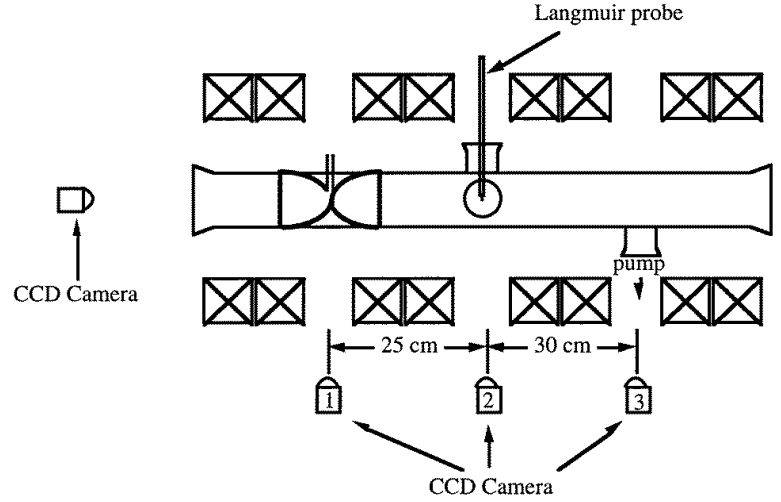

Figure 1. Schematic of the apparatus showing the various positions of the CCD camera.

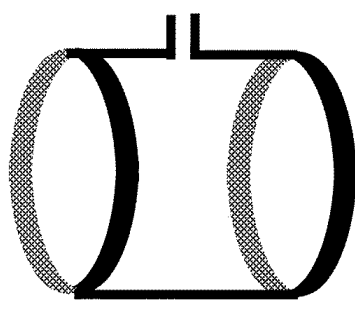

(a)

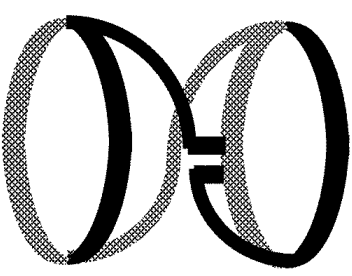

(b)
Figure 2. Geometry of the (a) Nagoya type III and (b) helical antennas.

between the RF amplifier and tuning circuit. Figure 2 shows the antennas used. The Nagoya type III antenna, figure 2(a), was $11 \mathrm{~cm}$ in diameter by $20 \mathrm{~cm}$ long, and the helical antenna, figure 2(b), was $11 \mathrm{~cm}$ in diameter by $16 \mathrm{~cm}$ long.

The end of the chamber nearer the antenna is sealed by a $10 \mathrm{~cm}$ diameter glass window to allow viewing of the plasma along the axis of the chamber. A Cohu Electronics type RS-170 CCD camera is positioned either in front of this window or at one of the three side ports (see figure 1) for transverse viewing. A bandpass optical filter with a central wavelength of $488 \mathrm{~nm}$ and a half-bandwidth of $10 \mathrm{~nm}$ is placed in front of the camera so that the only light collected is from $\mathrm{Ar}^{+}$emission, which is proportional to the square of the plasma density. The camera is focused at either the axial or radial centre of the chamber depending on its viewing direction. The camera has a shutter speed of 1/60 $\mathrm{s}$ and is triggered $20 \mathrm{~ms}$ after the RF pulse, when the plasma has reached steady-state conditions.

As will be discussed in section 4 , the $488 \mathrm{~nm}$ light intensity is proportional to $n^{2}\langle\sigma \nu\rangle$, where $n$ is the plasma density and $\langle\sigma \nu\rangle$ a steep function of electron temperature $T_{e}$. No RF-compensated probes were available this time for measuring $T_{e}$, but $2.5-3.5 \mathrm{eV}$ temperatures had been measured in a previous experiment on the same apparatus. To calculate $n$ from ion saturation currents, an average temperature of $3 \mathrm{eV}$ was used. The two-dimensional patterns seen with the CCD camera were therefore caused by a convolution of $T_{e}$ and $n$ variations, but these could not be separated because a $2 \mathrm{D}$ Langmuir probe drive was not available.
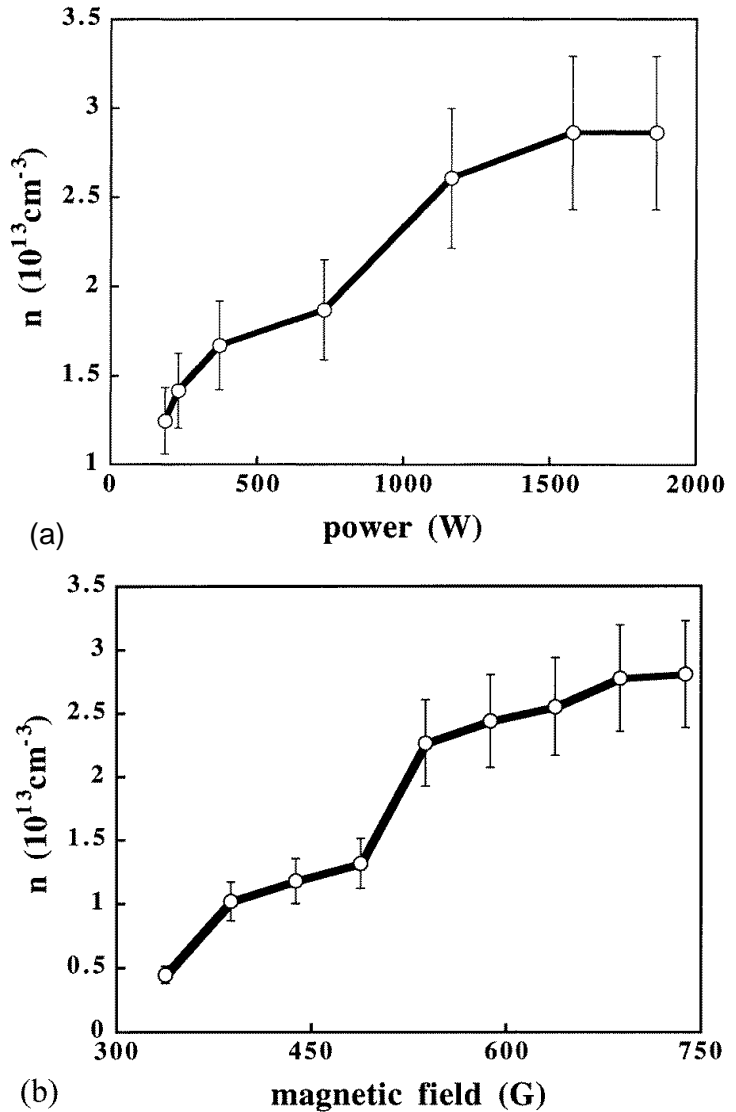

Figure 3. Argon plasma density at $3 \mathrm{mTorr}$ with the Nagoya type III antenna against (a) RF power at $740 \mathrm{G}$ and (b) magnetic field at $1.8 \mathrm{~kW}$.

\section{Results}

\subsection{Nagoya type III antenna}

The plasma density inferred from measurements of the ion saturation current versus input power $P_{r f}$ and magnetic field $B_{0}$ is shown in figures 3(a) and 3(b), respectively. The matching circuit was carefully retuned at each setting. These curves show more gentle 'jumps' as $P_{r f}$ and $B_{0}$ are raised than have been reported by others [5,7]. In the largest jump seen here, between 490 and $540 \mathrm{G}$ in figure 3(b), the plasma density increases by more than $40 \%$. The emission profile viewed along the cylindrical axis showed that during this increase the plasma was making a transition from being localized at two positions off axis to being somewhat bean shaped, with a higher concentration of light in the centre of the chamber (figures 4(a) and 4(b)). In both cases, the discharge is azimuthally asymmetric. At the highest magnetic field of $740 \mathrm{G}$, the plasma was observed to be concentrated along a line between the straight segments of the antenna, as seen in figure 5. Initially one could be inclined to think that these asymmetries were caused by the fact that the peak energy deposition for helicon waves is centred off axis [3], so that such a pattern of light could be explained perhaps by a plane-polarized wave excited by the antenna. To verify whether this distribution of light was due to a 


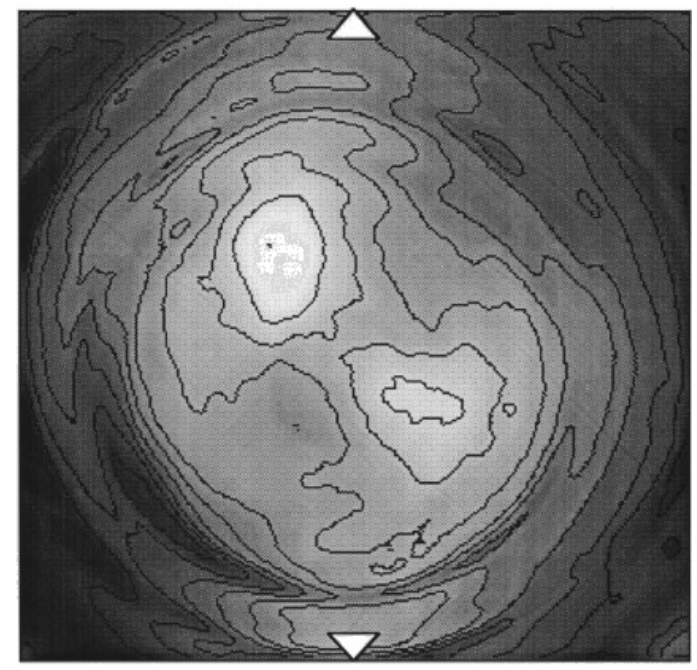

(a)

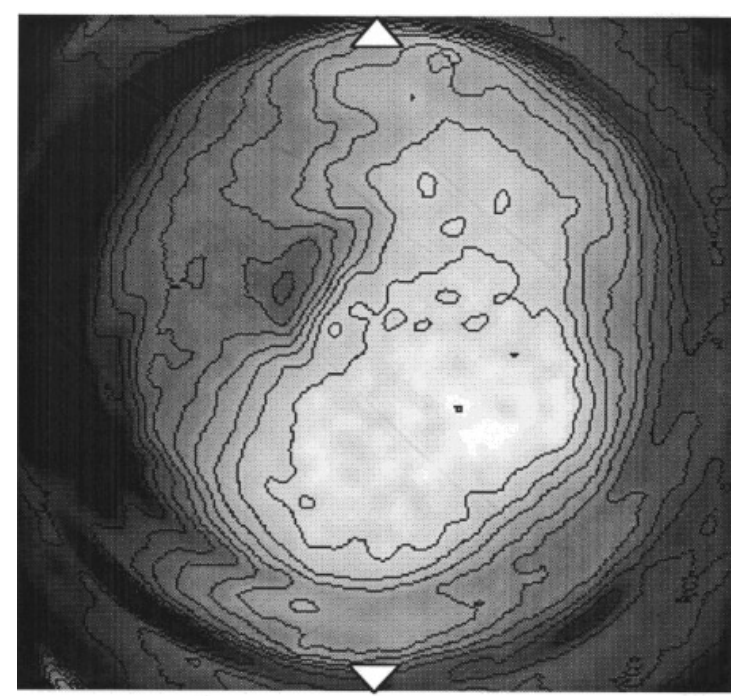

(b)

Figure 4. CCD images of the cross section of the plasma produced by the Nagoya type III antenna. Each contour denotes a $10 \%$ decrease in intensity. At approximately $500 \mathrm{G}$ magnetic field, the plasma density jumps abruptly as the transition from a predominantly capacitively coupled discharge to a partially inductively coupled one occurs. Immediately before the jump (a), most of the light is concentrated near the antenna legs, whose positions are shown by the triangles. After the jump (b), plasma is created in the centre of the chamber but is still asymmetric due to the influence of the antenna's electrostatic field. However, the onset of strong inductive coupling increases the light intensity by approximately a factor of two.

wave phenomenon, a Faraday shield was installed between the antenna and the glass chamber. The Faraday shield was a simple aluminium sheet with slots cut longitudinally and transversely as shown in figure 6 . This will shield the plasma from the electrostatic field generated by the high voltages on the antenna but will allow the electromagnetic fields generated by the antenna currents to pass through. After it was put in place, its effectiveness was tested using a capacitive probe designed similarly to that described

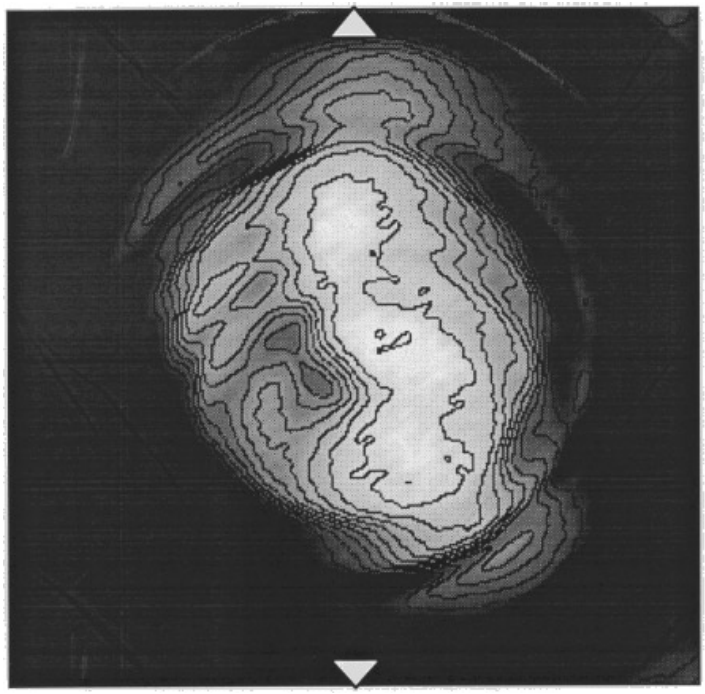

Figure 5. The plasma cross section for the Nagoya type III antenna at $740 \mathrm{G}$ and $1.8 \mathrm{~kW}$ of power, showing an elongation of the profile from top to bottom corresponding to the positions of the antenna wires.

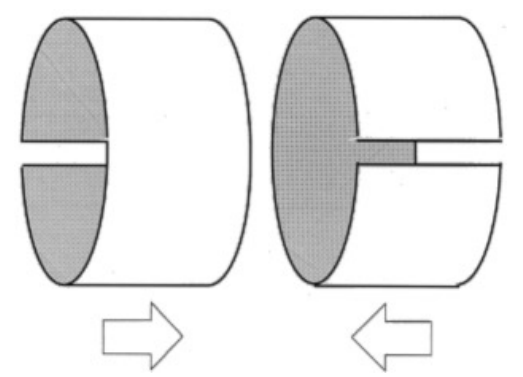

Figure 6. Geometry of the Faraday shield used to eliminate capacitive coupling from the antenna. The two sections have been separated in the drawing for clarity.

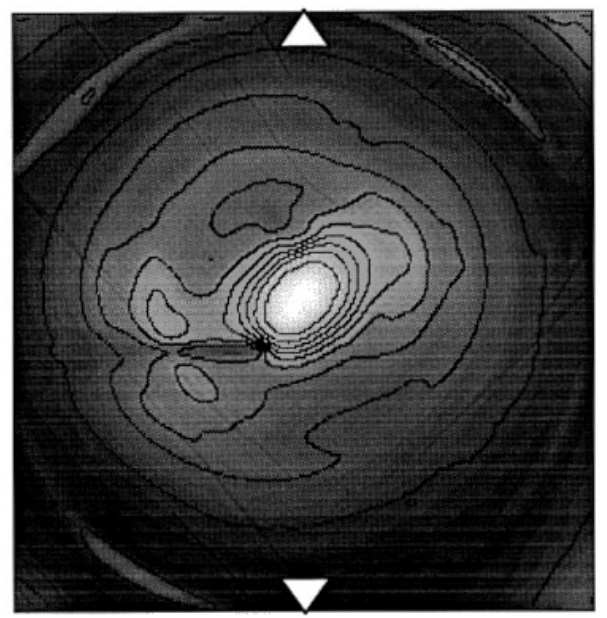

Figure 7. Same as figure 5, but with the Faraday shield installed. With the capacitive coupling eliminated, the plasma profile is nearly symmetric.

in [17] to verify that the vacuum electrostatic field was indeed being blocked. The electromagnetic field was also measured before and after installation in a similar manner 

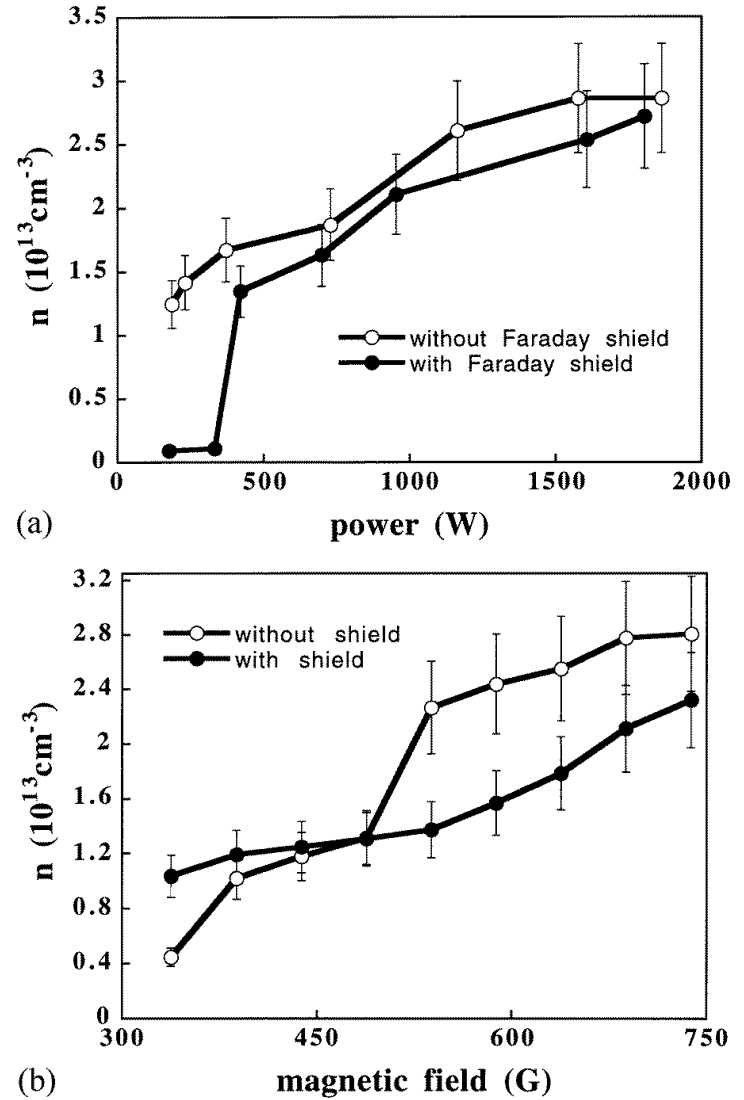

Figure 8. Plasma density with the shielded Nagoya type III antenna against (a) RF power and (b) magnetic field. Conditions same as in figure 3 .

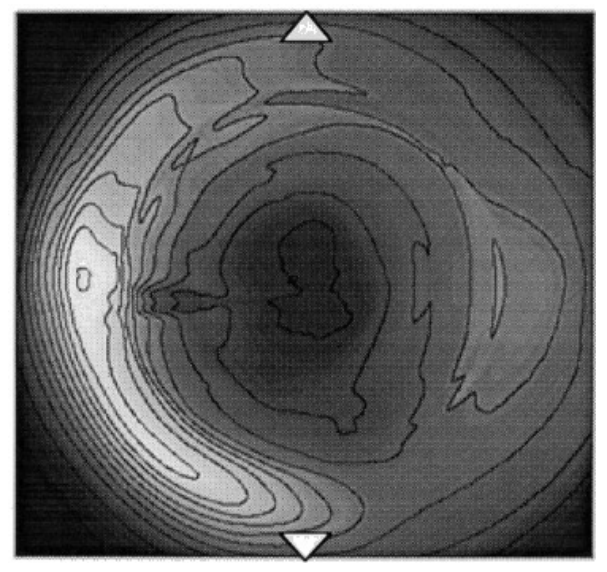

Figure 9. End view of the plasma in the low-power, low-density regime prior to the onset of helicon wave coupling at the density jump in figure $8(a)$.

using a magnetic loop probe as described in [6] to establish that that component of the field was minimally shielded.

With the shield installed, there were major differences in the discharge operation. The most noticeable was that the plasma emission profile was radically changed, becoming much more symmetric and centralized. This is demonstrated in figure 7, where the plasma image with the shield can be compared to the unshielded case of figure 5 .
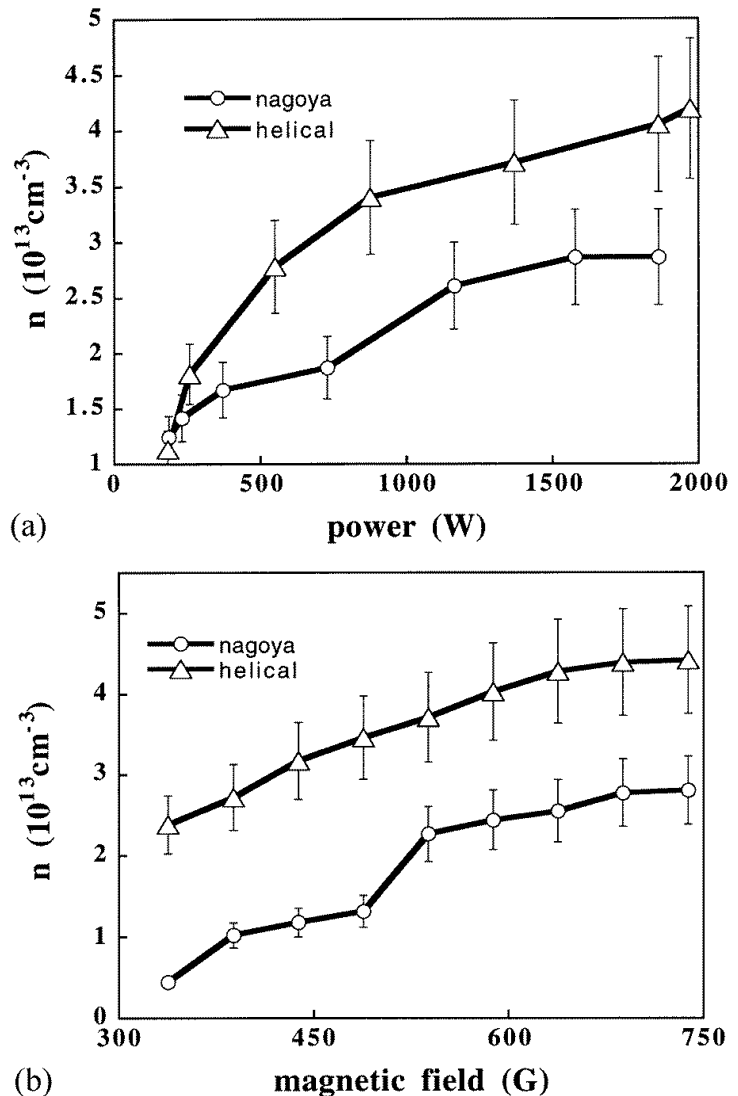

Figure 10. Plasma density with the helical antenna against (a) RF power and (b) magnetic field. The magnetic field and the antenna helicity combine to excite an $m=+1$ azimuthal mode. Other conditions same as in figure 3 .

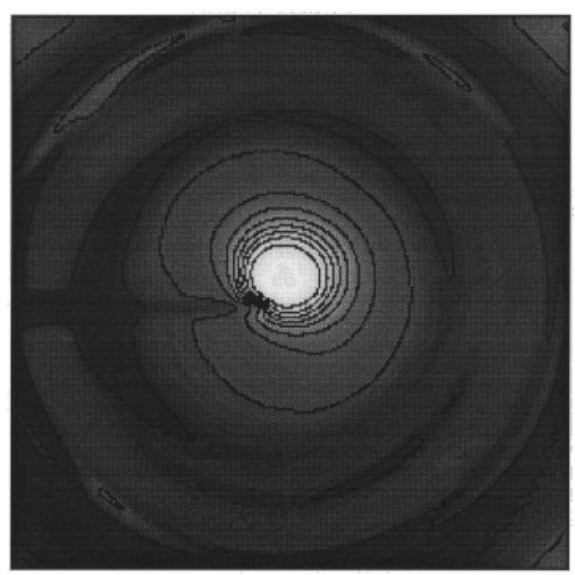

Figure 11. $\mathrm{Ar}^{+}$emission profile of the plasma produced by the helical antenna at $740 \mathrm{G}, 1.8 \mathrm{~kW}$ and 3 mTorr. A $10 \%$ filter was used, since the emission intensity in this image is $50 \times$ higher than with the Nagoya antenna under the same conditions (figure 5).

In fact, the sharp increase in density that was seen earlier in the range of $500 \mathrm{G}$ was no longer present once the shield was installed, as shown in figure 8. Also gone was the change in the plasma emission profile that accompanied this increase. However, a large drop in density now appears as the input power is reduced, an effect not observed when the 

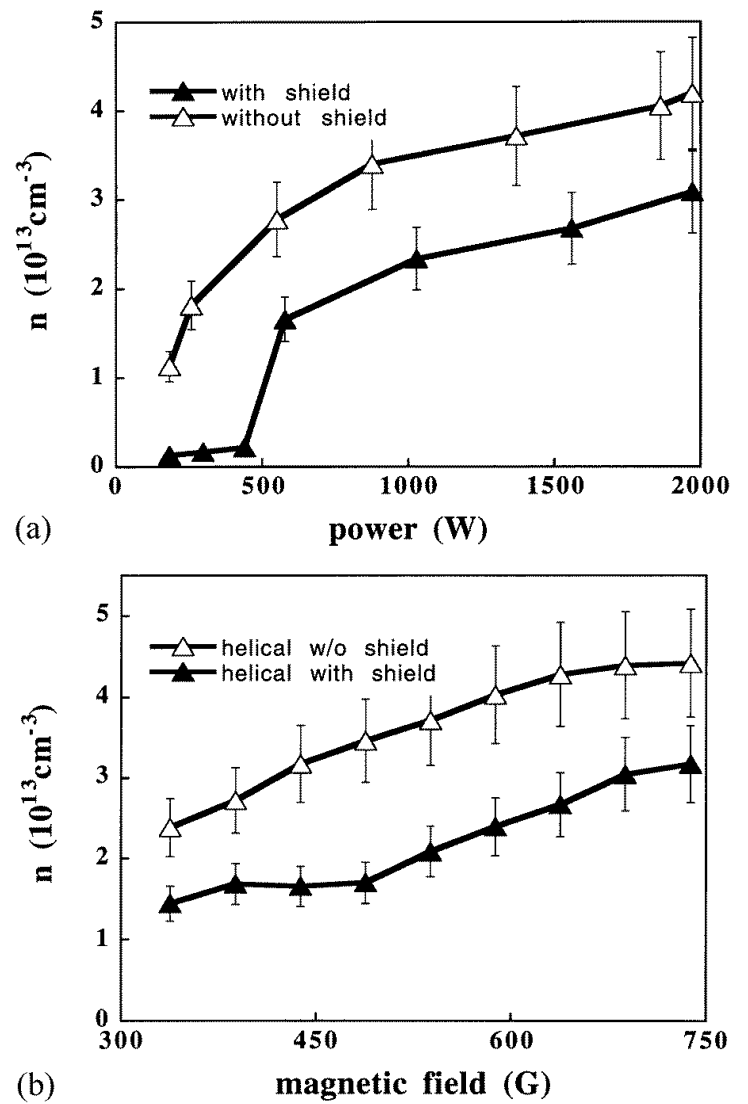

Figure 12. Plasma density with the helical antenna, with and without the Faraday shield, against (a) RF power and (b) magnetic field strength. Conditions same as in figure 3.

antenna was unshielded. Figure 9 shows that during this drop the plasma profile is again making a transition, this time from being centrally peaked to being edge localized. The asymmetry seen in figure 9 can be related to the location of the gap in the Faraday shield.

\subsection{Helical antenna}

Figures 10(a) and 10(b) show that, for the same values of power and magnetic field, the helical antenna always produces a higher peak plasma density than does the Nagoya type III. In addition, the emission profile for these discharges was always very symmetric azimuthally, as shown in figure 11 . This is not surprising, since the axial views are integrated over $z$, and the antenna has no preferred azimuth. The addition of the same Faraday shield used in our Nagoya-antenna measurements did nothing to change this profile, but did cause a density drop at low $P_{r f}$ similar to what we observed with the Nagoya antenna. This is shown in figure 12. Again, during this density drop the plasma profile changed from its normally very centrally peaked profile to an edge-localized profile similar to that of figure 9. Although the insertion of the Faraday shield did not change the symmetry of the discharge with the helical antenna, it did have the unexpected effect of decreasing the peak density by about a factor of two, as seen in figures 12(a) and 12(b). This could have been caused by partial shielding of the inductive field by currents in the Faraday shield that could flow around the single insulating gap. This simple shield was used because of the difficulty in eliminating ground loops and obtaining proper RF grounding. In fact, to obtain approximate settings for the tuning capacitors with the shield installed, it was necessary to inject electrons with an electron gun in order to obtain breakdown.

Another difference between the two antennas was that the plasma produced by the helical antenna was sensitive to one additional parameter: the direction of the magnetic field. Figure 13 shows transverse images of the plasma viewed at three axial positions for the three antenna configurations. Note that the plasmas produced by the helical antenna again are seen to have a distinct centre compared with the broad plasma produced by the Nagoya antenna. In the region farthest from the antenna, we see that the helical plasma with clockwise helicity relative to the magnetic field maintains its intensity and central structure. When the direction of the magnetic field is reversed, the plasma decays much more rapidly.

\section{Interpretation of results}

The optical emission at $488 \mathrm{~nm}$ can come from electron collisions with Ar I neutrals or with Ar II ions. Because of the higher threshold for direct excitation from Ar I, one would expect this process to be negligible. Indeed, in a previous paper Sudit and Chen [18] showed that data on the axial variation of $488 \mathrm{~nm}$ light agreed with calculations, based on measured $T_{e}$ values, assuming the $n^{2}$ dependence of the Ar II process but not with the $n$ dependence of the Ar I process. Thus, the light intensity in our CCD images should be proportional to $n^{2}\langle\sigma v\rangle$. The cross section $\sigma$ for excitation of the $488 \mathrm{~nm}$ line by electron impact on $\mathrm{Ar}^{+}$ ions was measured by Imre et al [19] and is shown in figure 14. Integrating this over a Maxwellian distribution gives the curve of $\langle\sigma v\rangle$ against $T_{e}$ shown by the dashed line in figure 15. Though this is a steep function of $T_{e}$, the latter varies little across the plasma because electron thermal diffusivity is large even across the magnetic field, a manifestation of what is commonly known as 'Langmuir's paradox'. This is shown by data taken with a fully RFcompensated probe [20] in another helicon experiment on the same apparatus under similar conditions. Figure 16 shows radial profiles of $T_{e}$ at $1 \mathrm{~kW}$ of RF power, 6 mTorr of $\mathrm{Ar}$ and various magnetic fields. The central densities are in the range $1.2-1.6 \times 10^{13} \mathrm{~cm}^{-3}$. It is seen that the profiles are nearly flat within the expected experimental error of $\pm 0.2 \mathrm{eV}$, with no systematic radial variation. The slight rise in $T_{e}$ at the edge is probably caused by the failure of RF compensation at densities below $10^{13} \mathrm{~cm}^{-3}$. The change in $\langle\sigma v\rangle$ when $T_{e}$ varies from 2.5 to $3.5 \mathrm{eV}$ is shown by the shaded portion of the $\langle\sigma \nu\rangle$ curve in figure 15 and amounts to a little more than an order of magnitude. By comparison, the variation in the $n^{2}$ factor is shown in figure 15 as the solid curve, and this also varies by a little more than an order of magnitude as $n$ varies from 1 to $5 \times 10^{13} \mathrm{~cm}^{-3}$, as it does in the density data given in this paper. Thus, the light patterns are attributable to both $T_{e}$ and $n$ variations during 

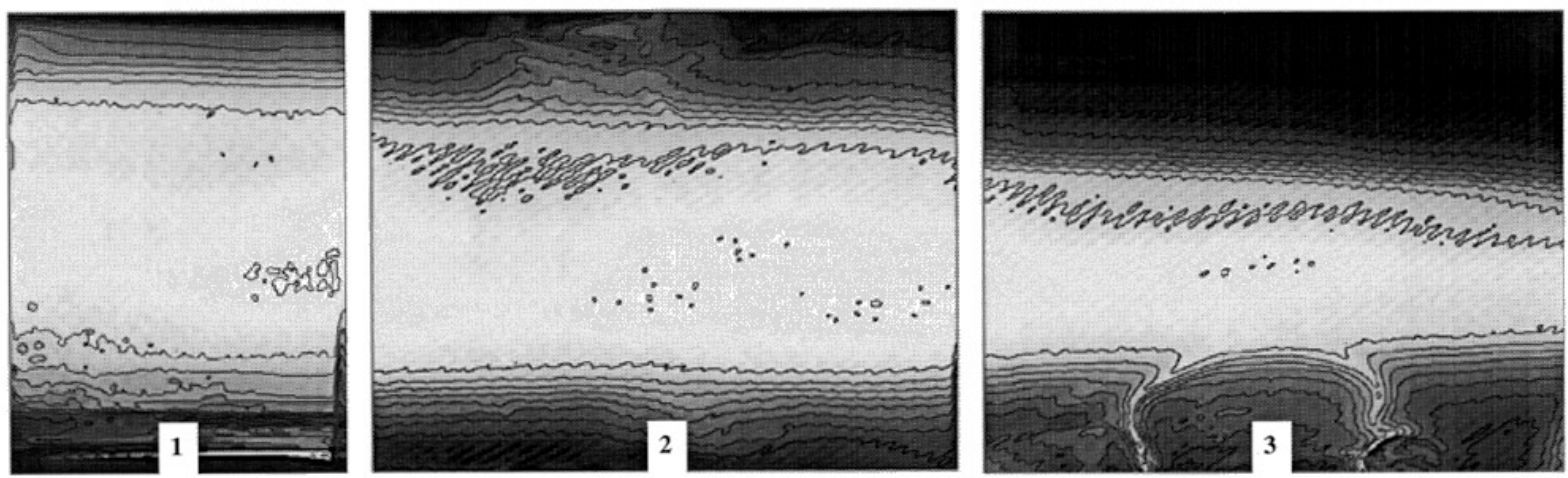

(a)
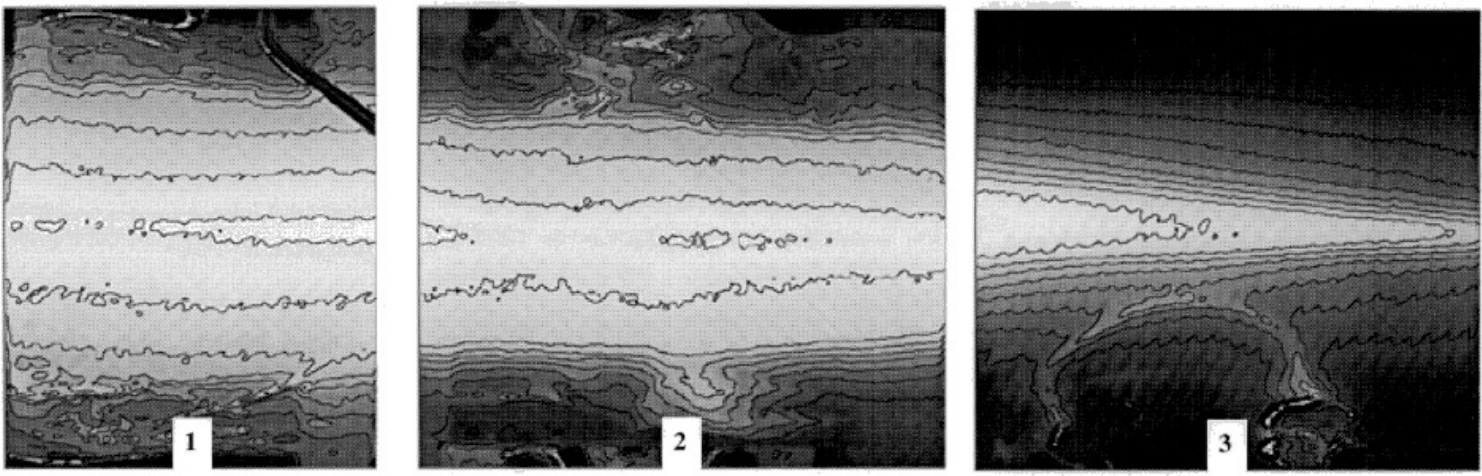

(b)
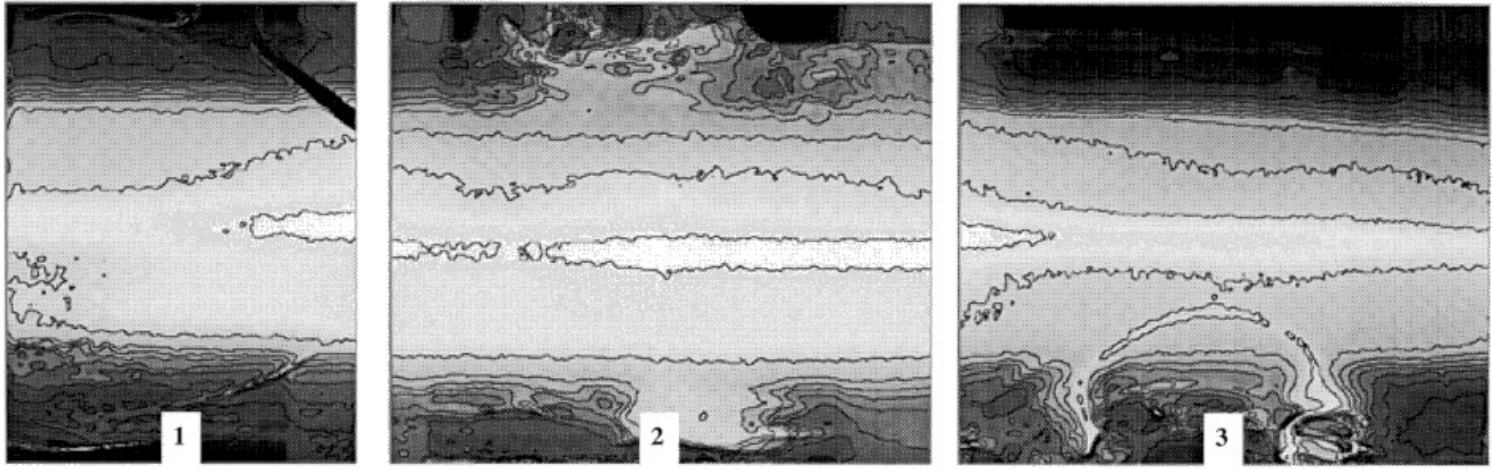

(c)

Figure 13. Transverse image of the discharge at $740 \mathrm{G}$ for the three antenna configurations: (a) Nagoya type III, (b) left-handed helical and (c) right-handed helical. The numbers shown correspond to the three camera positions in figure 1. The brightest and longest plasma (c) corresponds to the $m=+1$ azimuthal mode.

jumps from capacitive coupling to inductive coupling and finally to helicon wave generation.

Because of the $17 \mathrm{eV}$ threshold in figure 14, optical emission is extremely sensitive to high-energy tails in the electron distribution. If such hyperthermal electrons exist in large numbers, they would be responsible for the observed light patterns. Our conclusions that the plasma changes in a two-dimensional manner when abrupt jumps in axial density are observed or when Faraday shields are installed are nonetheless valid. We do not believe, however, that hyperthermal electrons abound in helicon discharges at densities well above $10^{13} \mathrm{~cm}^{-3}$. In a previous experiment [21], a carefully RF-calibrated gridded energy analyser was used, and no electron tails could be seen down to the measurement limit of $1 \%$.

\section{Discussion}

From the images of the plasma observed with and without the Faraday shield installed, it is clear that the asymmetries observed in the light emission profile cannot be solely attributed to wave coupling phenomena. Even at very high densities and magnetic field strengths, where the discharge has been described as being primarily wave driven, figures 8 and 12 demonstrate that a significant amount of power is capacitively coupled. Although it is likely that the inductive 


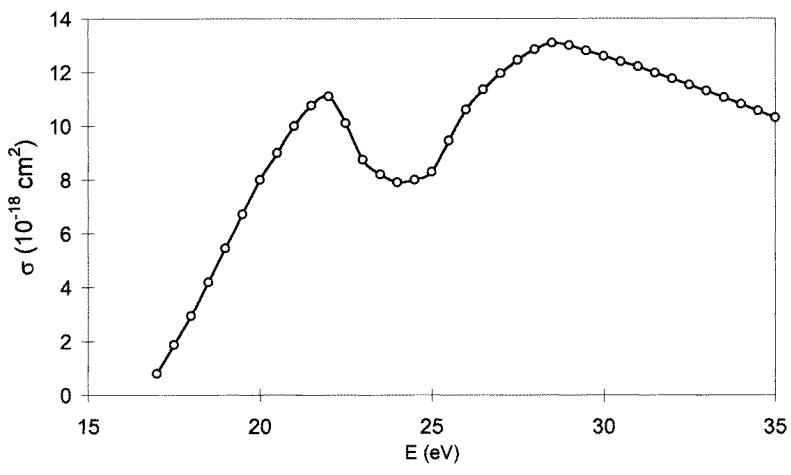

Figure 14. Cross section for excitation of the $488 \mathrm{~nm}$ line in $\mathrm{e}-\mathrm{Ar}^{+}$collisions as a function of electron energy.

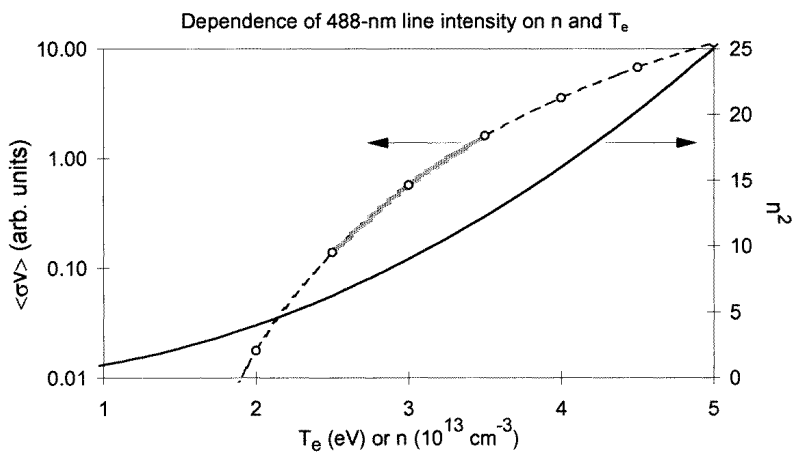

Figure 15. Computed variation of the probability of exciting the $488 \mathrm{~nm}$ line from $\mathrm{Ar}^{+}$due to changes in electron temperature (dashed line) and changes in density (solid line). The expected range of $T_{e}$ in the experiment is shown by the shaded portion of the dashed line, while the range of densities in the experiment covers the entire graph.

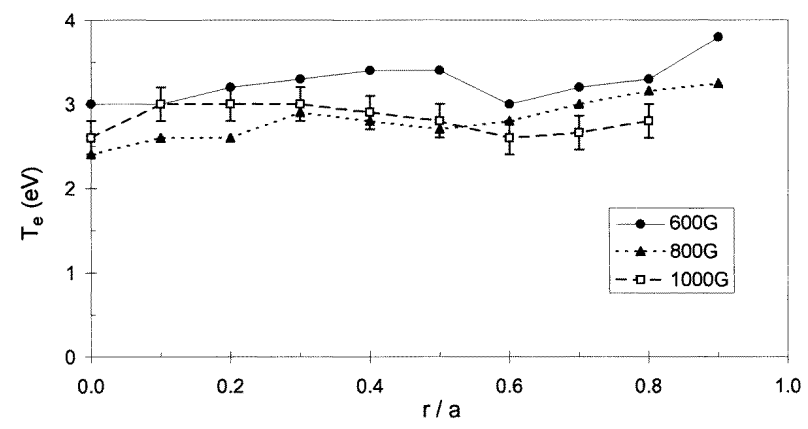

Figure 16. Radial profiles of electron temperature in similar helicon discharges studied earlier.

coupling is the process responsible for the most of the heating of the higher-density plasmas, a complete picture of the power deposition and antenna coupling must include both components.

Recall that there was an abrupt decrease in plasma density and a transition in the emission profile that occurs in the range of $500 \mathrm{~W}$ when the antennas were shielded. This can be explained as follows. When the antenna is initially turned on, capacitive coupling is needed to provide initial breakdown of the gas. Once a plasma has developed, the

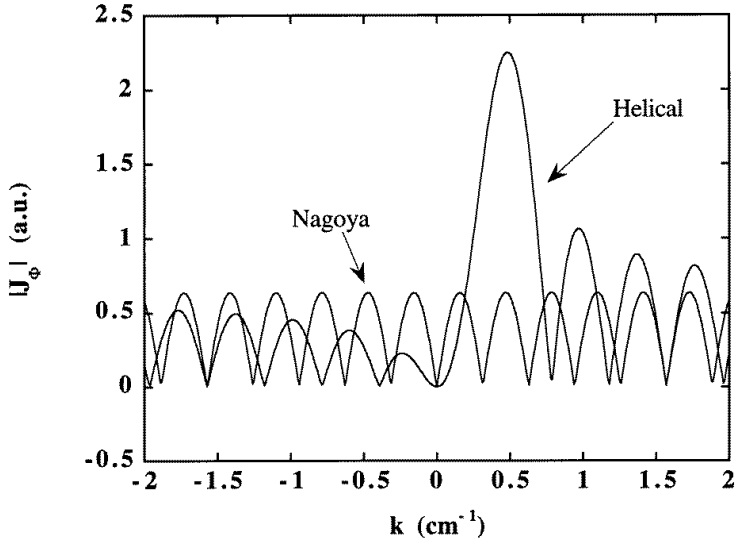

Figure 17. The $k$-spectra of the antenna current $J_{\varphi}$ for the helical and Nagoya type III antennas.

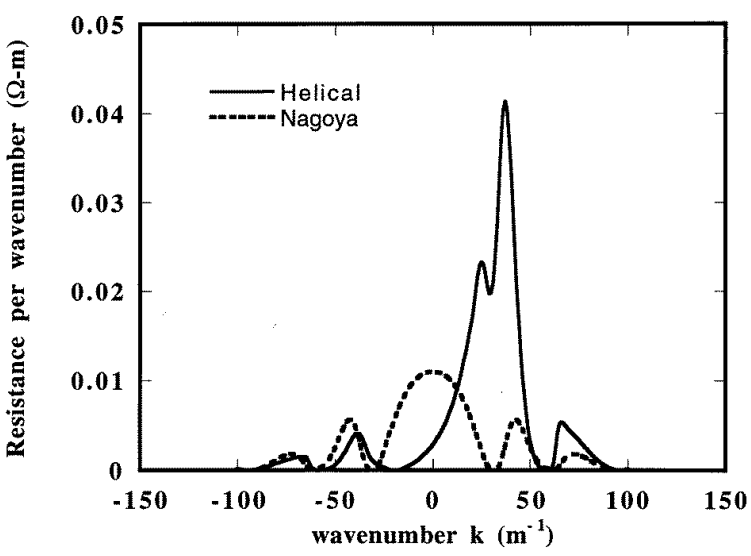

Figure 18. The $k$-spectra of antenna loading resistance calculated for both types of antenna with a mean plasma radius of approximately $3 \mathrm{~cm}$, a peak density of

$3 \times 10^{13} \mathrm{~cm}^{-3}$ and a magnetic field of $800 \mathrm{G}$. The helical antenna spectrum is seen to be asymmetric with much higher loading than for the Nagoya type III antenna.

inductive fields can heat it, and the density will rapidly increase. There is a minimum initial density required for this inductive heating to be effective.

This minimum density is a consequence of the fact that the inductive fields have a spatial structure and dispersion relation imposed on them by the plasma. These are what are referred to as the plasma eigenmodes. Because of these eigenmodes, only a fraction of the inductive fields of the antenna have the correct structure to be supported by the plasma. The larger this fraction is, the more inductive power can be utilized to heat the plasma. This is why the antenna geometry is important and why the helical antenna is better coupled than the Nagoya type III.

A rigorous treatment of this problem has been done by Shamrai and Taranov [13], in which they calculate the linear response of a uniform plasma to an external antenna current. More recently Arnush and Chen [15] have generalized this calculation to a nonuniform plasma. In both calculations the amplitude of the induced fields in the plasma is found to be related to the Fourier transform of the antenna current 
by

$$
\Psi_{i}=F_{i}(r, m, k) J_{\varphi}(m, k) .
$$

Here $\Psi_{i}$ is the amplitude of a field component ( $\boldsymbol{E}$ or $\left.\boldsymbol{B}\right)$, $F_{i}$ is a function of the plasma parameters (e.g., density, magnetic field and radial profile), and $J_{\varphi}$ is the Fourier transform of the antenna surface current, defined as

$$
J_{\varphi}(m, k)=\frac{1}{2 \pi} \int_{-\infty}^{\infty} \mathrm{d} z \int_{0}^{2 \pi} \mathrm{d} \varphi J_{\varphi}(\varphi, z) \mathrm{e}^{-\mathrm{i}(m \varphi+k z)} .
$$

For the helical antenna, this term is

$$
J_{\varphi}(m, k)=\frac{k}{m \pi} \frac{\sin \left(\frac{1}{2} k L-m \theta\right)}{\frac{1}{2} k L-m \theta}
$$

while for the Nagoya type III antenna it is

$$
J_{\varphi}(m, k)=\frac{2}{m \pi} \sin \left(\frac{k L}{2}\right) .
$$

Here $L$ is the length of the antenna, $m$ is the azimuthal mode number and $\theta$ is the pitch angle of the helix for the helical antenna. Figure 17 shows that the spectrum of the helical antenna is narrower and has a larger amplitude than that of the Nagoya type III antenna for the lowest azimuthal modes $m= \pm 1$. In addition, as shown in [15], the plasma response is invariant on reversing the sign of $k$ but not on reversing the sign of $m$, so that the azimuthal mode content will be generally be more heavily weighted towards either clockwise or counterclockwise polarization. Experimentally, this was demonstrated in figure 13 by reversing the magnetic field, which amounts to changing $k$ to $-k$ in (3). The spectral power density for each antenna was calculated numerically by solving the boundary value problem as outlined by Arnush and Chen [15]. This can be expressed as the antenna loading resistance, where the resistance is related to the inductive power deposited by Ohm's law:

$$
P=\frac{1}{2} I_{a}^{2} R
$$

where $I_{a}$ is the antenna current. The higher the resistance, the more efficient the antenna configuration, since the sameamplitude waves can be driven using a smaller amount of current. In practice the total load to the powers supply will also include losses in the circuit and capacitive loading, so that the total power used will be

$$
P=\frac{1}{2} I_{a}^{2}\left(R_{i}+R_{c}+R_{s}\right)
$$

with $R_{i}$ representing the inductive (wave) loading, $R_{c}$ the non-inductive (capacitive) loading and $R_{S}$ the stray losses in the circuit. Experimentally, the best helicon wave coupling is obtained by designing the antenna with $R_{i}$ as large as possible. Figure 18 shows the loading resistance per wavenumber, with the integrated area under each curve representing the total loading resistance $R$. We can see that the loading spectrum for the helical antenna is not only much narrower for the $m=+1$ mode, but the loading resistance is also an order of magnitude larger than for any other antenna configuration.

\section{Summary}

We have seen that the role of capacitive coupling from the antenna is in general a non-negligible effect and should be considered as an important part of any power calculations. In addition, depending on the antenna geometry, this coupling can have a large affect on the observed light emission from the plasma. The inductive component of the antenna apparently couples to a symmetric, centrally peaked plasma. Antenna configurations which produced the highest-density plasmas were numerically calculated to have the highest antenna loading, with the $m=+1$ configuration having the largest loading and producing the longest, brightest plasma. This strongly supports the notion that the helicon waves are not simply incidental in a highdensity discharge but are responsible for producing the discharge.

\section{Acknowledgments}

This work was supported by the National Science Foundation, grant No ECS-9400849, the Semiconductor Research Corporation, project No IJ-529, and the Wisconsin Engineering Center for Plasma-Aided Manufacturing. We thank Professor Donald Arnush for considerable help with the interpretation of the results.

\section{References}

[1] Boswell R W 1984 Plasma Phys. Control. Fusion 261147

[2] Boswell R W, Porteus R K, Prytz A, Bouchoule A and Ranson P 1982 Phys. Lett. 91A 163

[3] Chen F F and Chevalier G 1992 J. Vac. Sci. Technol. A 10 1389

[4] Komori A, Shoji T, Miyamoto K, Kawai J and Kawai Y 1991 Phys. Fluids B 3893

[5] Degeling A W, Jung C O, Boswell R W and Ellingboe A R 1996 Phys. Plasmas 32788

[6] Light M and Chen F F 1995 Phys. Plasmas 21084

[7] Shoji T, Sakawa Y, Nakazawa S, Kadota K and Sato T 1993 Plasma Sources Sci. Technol. 25

[8] Davies B J 1970 J. Plasma Phys. 443

[9] Woods L C 1962 J. Fluid Mech. 13570

[10] Ellingboe A R and Boswell R W 1996 Phys. Plasmas 3 2797

[11] Chevalier G and Chen F F 1993 J. Vac. Sci. Technol. A 11 1165

[12] Fischer B, Kraemer M and Enk Th 1994 Plasma Phys. Control. Fusion 362003

[13] Shamrai K P and Taranov B V 1996 Plasma Sources Sci. Technol. 5474

[14] Kamenski I V and Borg G G 1996 Phys. Plasmas 34396

[15] Arnush D and Chen F F 1997 Phys. Plasmas submitted

[16] Kim J H, Yun S M and Chang H Y 1996 IEEE Trans. Plasma. Sci. PS-24 1364

[17] Savas S E and Donohoe K G 1989 Rev. Sci. Instrum. 60 3391

[18] Sudit I D and Chen F F 1996 Plasma Sources Sci. Technol. 543

[19] Imre A I, Dashchenko A I, Zapesochnyi I P and Kel'man V A 1972 JETP Lett. 15503

[20] Sudit I D and Chen F F 1994 Plasma Sources Sci. Technol. 3162

[21] Blackwell D D and Chen F F 1996 Proc. SRC Techcon '96 (Phoenix, AZ, 1996) (Semiconductor Research Corporation) 